\title{
Estimating Contextual Motivating Factors in Virtual Inter-organizational Communities of Practice: Peer Effects and Organizational Influences
}

\begin{abstract}
Virtual inter-organizational communities of practice (IOCoPs) enable professionals belonging to different organizations to exchange and share knowledge via computer-mediated interactions. Since knowledge sharing is socially embedded, contextual factors likely play an important role in encouraging individuals' community participation. Specifically, professionals in IOCoPs are embedded in two different social environments: the virtual community where they interact with online peers and organizations where they utilize their knowledge. Therefore it is important to simultaneously study motivating factors generated from these two different contexts, including peer effects within and organizational influences outside the virtual community. In this research, we apply a novel econometric identification method to analyze a unique dataset collected from a virtual IOCoP in the financial trading sector. We find that, after controlling for individual level characteristics, contextual motivating factors from peers and organizations are influential both quantitatively and qualitatively in determining community participation. Differentiating multiple-level motivating factors across different contexts enables us to shed light on various mechanisms that IOCoPs can apply to engage collective learning and knowledge management across organizations.

Keywords: inter-organizational communities of practice, peer effects, organizational influences, multilevel framework

\section{Introduction}

Knowledge is a key organizational asset that sustains a firm's competitive advantages. Understanding the creation, mobilization, and management of knowledge has been an enduring research theme in the management literature (Majchrzak et al. 2013; Hass et al. 2015). Most organizations do not possess all required knowledge within their formal boundaries; thus, they need to acquire knowledge from external organizations and individuals (Wasko and Faraj 2005). Inter-organizational Communities of Practice (IOCoPs) represent one critical channel for accessing external expertise. These communities bring
\end{abstract}


together professionals from different organizations, allowing for them to exchange ideas, share knowledge, and learn from each other (Wenger et al. 2002). Such communities have proliferated in recent years due to the widespread use of information technologies that facilitate increased knowledge creation and sharing. For instance, music teachers from different schools have formed IOCoPs to advance effective pedagogy within their school districts (Battersby and Verdi 2015). Similar communities have also been organized to facilitate collaborative professional development among police officers, public health practitioners, solid waste managers, IT developers, and professionals from many other industries (Cheung et al. 2013).

IOCoPs provide an important tool for knowledge management and professional development (Richard et al. 2014), and are characterized by certain key features. First, the identity of the community is defined by a field of professional knowledge, and the community's sustainability depends on the exchange of knowledge between participants. Second, community participants are a group of professionals with a shared practice and understanding, who work and learn together to pursue common goals. Third, those participants come from different organizations, and they also apply knowledge shared and learned from the community back to their organizations. It is not uncommon that participants in IOCoPs reveal their true personal and institutional identities in the community. Therefore, IOCoPs embrace the interplay between virtual and real-world facets of a group of professionals, and the interplay between these individuals and the organizations they belong to. Due to these special features of the IOCoP, participants are simultaneously embedded in two different social environments: the virtual community itself and organizations these participants are affiliated with. Therefore, it is important to carefully disentangle contextual motivating factors from online peers within the community as well as these individuals' work environment. A multilevel framework focusing on interrelationships among individuals, network structures, and institutions is needed in order to provide a deeper and richer portrait of this form of digitally mediated collaboration (Sarker and Valacich 2010).

Within the community, experience-based knowledge is exchanged and combined via user interactions (Brown and Duguid 1991). In addition to social norms formed and shared among all community 
participants, individuals are also subject to potential influence from peers they directly communicate with in the community. Online peers are those who either answer a question of or ask a question to a focal individual. There are two types of peer effects, as peers can influence a focal individual endogenously through their behavior (i.e., what they do) and exogenously through their characteristics (i.e., who they are) (Manski 1993). However, subtle differences between endogenous and exogenous peer effects have not been explored in-depth in virtual communities.

Within their affiliated organizations, individuals not only apply knowledge obtained from virtual IOCoPs in their work environment, but also bring back their organizational knowledge into virtual communities. It is well known that organizations play a key role in articulating and amplifying knowledge developed by individuals (Nonaka 1994). However, it is not clear how organizations shape individuals’ knowledge sharing and consumption outside their formal boundaries. Some argue that organizational characteristics provide context for individuals, and they can affect individual attitudes and behavior as a higher-level situational factor (John 2006). Others suggest that individuals could ignore contextual forces, and "the presence of contextual variables does not mean they will shape behavior" (Mowday and Sutton 1993, pp. 209). Thus, it is intriguing to explore whether individuals are still influenced by organizations they are working for in virtual IOCoPs, where participation is purely autonomous and voluntary.

Our research aims to enhance current understanding of participation motivations in virtual communities in several ways. First, empirical studies that cross levels of analysis remain relatively novel (Sarker and Valacich 2010) and warrant attention (Faraj et al. 2016). Using a rich and unique dataset from a virtual IOCoP on financial information exchange protocols, we theorize and empirically estimate the incentives to participate and contribute in virtual IOCoPs from a true multilevel perspective. It is also one of the first to study impact of social media in electronic financial trading, an industry with a $\$ 55$ trillion market size (Perry 2013). Second, due to special features of IOCoPs, we identify and differentiate contextual factors generated from two different social environments, where knowledge flows occur. Third, our study represents, to the best of our knowledge, the first attempt to distinguish endogenous and exogenous peer effects in virtual communities. It helps us better understand whether and how online peers 
influence each other. Fourth, organizational attributes examined in this study are observable to individual employees. We examine their influences even without requiring those organizations to actively intervene in their employees' IOCoP participation.

Our analysis yields several interesting results about contextual motivating factors from online peers and organizations. We find evidence for both endogenous and exogenous peer effects in our virtual IOCoP. The existence of endogenous peer effects suggests the positive spillover effects of individuals' efforts to participate in the virtual environment. At the same time, exogenous peer effects indicate that individuals' characteristics also matter to their online peers. For instance, our results highlight the vital role of long time participants. Individuals' seniority not only is positively associated with their own community participation level, but also encourages their peers' participation. At the organizational level, individuals' online behavior outside of organizational boundaries is still subject to influences from their work environment. More interestingly, our results suggest that organizations and virtual IOCoPs complement each other as two learning channels. If individuals come from more knowledgeable organizations, they participate less quantitatively in the virtual IOCoP, but their contribution quality is higher.

The remainder of this paper is structured as follows. Section 2 briefly reviews related literature on motivating factors in online communities in order to identify the research gap. Section 3 develops hypotheses and section 4 describes our empirical setting and data used for econometric analyses. Section 5 is devoted to model development. Section 6 presents results and analyses. Section 7 summarizes our findings, contributions to theory development, as well as the managerial implications of knowledge creation and dissemination in an open technology-enabled platform.

\section{Assessment of Research Gap in Prior Literature}

Despite the extensive literature on incentives to participate and contribute in virtual communities (e.g., Constant et al. 1996; Shah 2006; Wasko and Faraj 2005; Xia et al. 2012), relatively limited attention has been paid to examine contextual motivating factors from both the peer and organizational levels. Thus we mainly focus on reviewing closely related literature at these two levels. 
Existing studies that have analyzed peer influences on individual behavior mainly consider aggregated influences from all other participants in the community. For example, Zhang and Zhu (2011) find that shrinking group size reduces social benefits, which negatively affects individuals' contribution levels in Chinese Wikipedia. Ahn et al. (2014) suggest that individuals' contribution depends on whether they believe others will engage as well. Tsai and Bagozzi (2014) demonstrate that users are encouraged to contribute more in virtual communities if they accept group norms and conform to other group members. However, aggregating all other participants' influences may overlook the structure of the underlying social network within the community. A focal individual communicates directly with her directly connected peers, so it is likely that these peers play a more important role in shaping the focal individual's behavior than other participants in the community. In other words, social influence tends to be "local" (Sundararajan 2008). Recent studies have taken into consideration of social network structure to analyze online user behavior (Aral 2011). For instance, Zeng and Wei (2013) study photo uploading between the dyads of Flickr users. An online user typically has more than one friend, so our study examines influences from all peers a focal user interacts with. Shriver et al. (2013) study how friend requests affect individuals' blogging behavior and find that friendship formation positively affects the creation of usergenerated contents. Compared with Shriver et al. (2013), we explore whether peers' behavior and characteristics influence an individual's participation after social ties are established. The experimental method has been introduced to examine peer effects on product diffusion in online networks (Bapna and Umyarov 2015). Our study only has multiyear observation data collected from a virtual IOCoP, and we adopt the extended linear-in-means estimation framework proposed by Bramoulle et al. (2009) in order to identify both the endogenous and exogenous peer effects. While the new approach has been used to empirically estimated peer effects in offline settings (Bramoullé et al 2009; De Giorgi et al. 2010; Pool et al. 2014), its application in the virtual world is rather scarce. In this paper, we extend Bramoullé et al. (2009) to analyze panel data and accommodate fixed effects accounting for unobservable factors at the individual level. Furthermore, the subtle differences between endogenous and exogenous peer effects have not been fully recognized and investigated in virtual communities. 
Virtual community research on organizational level influences is limited, as work-related communities are fewer than their non-work related counterparts (Spaeth et al. 2015). Constant et al. (1996) study the enterprise email system at a global computer manufacturer. They identify several incentives provided by a company for the virtual community formed within the organization, such as being good organizational citizens. Ardichvili et al. (2003) investigate virtual knowledge-sharing communities of practice at a Fortune 100 company. They also suggest that a supportive organizational culture and various types of trust developed within an organization are critical for encouraging individuals to participate in virtual CoPs. Jeppesen and Frederiksen (2006) also examine firm-hosted virtual communities. It remains unclear whether an organization can exert similar influences in virtual IOCoPs, which are communities located externally, with no formal relationship to the organization. Open source software (OSS) literature has explored how organizations can be actively involved in OSS communities, using strategies such as monetary incentives, sponsorship, and community-based credibility and openness (Roberts et al. 2006; Shah 2006; Spaeth et al. 2015). In this research, we follow this line of research to explore additional organizational level attributes that could affect the employees' participation in virtual IOCoPs. Specifically, we study observable organizational attributes in order to understand their impacts, even when organizations may not explicitly direct or command their employees' community activities.

While not being our main focus, individual level incentives are controlled in the study. Motivating factors at the individual level have been researched extensively and we classify them into three main categories. The first is the capability-based perspective, which considers whether individuals have the ability to contribute. Examples include cognitive capital or tenure (Shriver et al. 2013; Majchrzak et al. 2013; Tsai and Bagozzi 2014). The second is the utility-based perspective, which identifies various benefits offered by the community that are valuable to individuals. For instance, individuals can learn new knowledge, earn respect, advance their careers, or simply enjoy community activities (Ahn et al. 2013; Hass et al. 2015). The third is the pro-social perspective, where individuals contribute in order to benefit others, the community, or the society as a whole (Constant et al. 1996; Wasko and Faraj 2005; Xia et al. 2012). 


\section{Hypotheses Development}

Based on extensive literature review and data available for our analyses, we propose hypotheses of contextual factors while controlling motivating factors at the individual level. To accurately depict an individual's behavior in a virtual IOCoP, we measure the level of participation both quantitatively and qualitatively. In terms of participation quantity, we differentiate knowledge consumption from knowledge contribution, as these two distinct types of knowledge sharing are both desirable (Kankanhalli et al. 2005; Ridings et al. 2006) and represent the supply and demand sides of knowledge, respectively. In addition, we assess quality of knowledge contributed by an individual, which is another important measure of community participation (Wasko and Faraj 2005).

\subsection{Organizational Influences}

Knowledge exchanged in virtual IOCoPs is used for job-related activities and as a way to increase individuals' marketability. Since knowledge utilization happens in the organization, we hypothesize that the contexts of the organization shape individuals' behavior of accessing and creating such knowledge in virtual IOCoPs (Mowday and Sutton 1993; Spaeth et al. 2015). Prior studies have identified various sponsorship mechanisms organizations can apply to encourage participation of their employees in OSS projects (Shah 2006; Spaeth et al. 2015). In our research, organizations are not directly and actively involved in IOCoP activities. However, we argue that certain organizational attributes can still signal organizations' commitment and identification towards the IOCoP, and consequently affect the enthusiasm of their employees.

The first organizational attribute identified in our research is organizations' consortium membership, which indicates whether organizations support the common goal of the IOCoP. The virtual IOCoP we study in this paper discusses messaging standards for electronic financial asset trading. The standards are developed and managed by a non-profit and industry-driven standard consortium in the financial service industry, FIX Trading Community. As a neutral and independent industry association, FIX Trading Community is open for any organization that is interested in becoming a consortium member to influence and promote the development and adoption of its standards. Compared with non-member organizations, 
member organizations have stronger beliefs in the value of the standards, and identify more with the shared mission to enhance interoperability for global financial trading (Zhao et al. 2011b). In other words, consortium membership status distinguishes organizations in terms of their commitment and support towards the field of professional knowledge exchanged in the IOCoP. Such organizational attribute is compatible with individuals' motivation to participate in the IOCoP for several reasons. First, individuals tend be more enthusiastic about the standards if their organizations have sponsored the standard consortium. The IOCoP offers them valuable learning opportunities to learn more about the standards. Second, individuals from member organizations are more likely to develop a stronger sense of identity and belonging in the virtual IOCoP organized and hosted by the consortium. Ongoing sense making happens in individuals' organizational life (Bloor and Dawson 1994), and individuals from member organizations tend to share their organizations' beliefs and commitment towards the standard consortium. Such a sense of community encourages individuals to participate more in virtual communities (Blanchard and Markus 2004).

H1: The level of participation in the virtual IOCoP is higher for a focal individual from a consortium member organization than that of an individual from a non-member organization.

The second organizational attribute is organizational knowledge, which is distinct from, yet interdependent of, individual knowledge (Bhatt 2002). Organizations possess knowledge in their routines and repositories (Nelson and Winter 1982). They create knowledge through socialization, combination, externalization, and internalization (Nonaka 1994; Hass et al. 2015). An organization can deepen its employees' knowledge through formal job-specific training as well as informal social interactions among employees. Some knowledge is informal, situated, experience-based, and can be effectively exchanged and combined via social interactions (Lave and Wenger 1991; Spaeth 2015). If an organization has more individuals possessing diverse expertise, its employees have better chances to collaborate, share knowledge with one another, and use a collective learning approach to empower themselves. Individuals participate in the IOCoP in order to exchange knowledge with online peers from other organizations. Their organizational knowledge better equips them to contribute to the virtual community. Those 
individuals also have more co-workers at work to discuss knowledge obtained from the IOCoP. Therefore, we hypothesize that:

\section{H2: A focal individual's level of participation in a virtual IOCoP is positively related to her} organization's knowledge.

\subsection{Peer Effects}

By joining research from both sociology and economics, social economics recognizes the importance of social interactions in shaping individual behavior and group outcomes (Durlauf and Young 2001; Bramoullé et al. 2014). Individual characteristics alone cannot fully explain aggregated behavior in groups, such as fashion (Bikhchandani et al. 1992), obesity (Trogdon et al. 2008), and participation in retirement plans (Saez and Duflo 2003), due to the dependence of an individual's choice on the choice of others (Soetevent 2006). For instance, adolescent weight gain at the individual level is related to the weight of their peers (Trogdon et al. 2008).

It is quite challenging to empirically identify peer effects, despite their importance. The reason is that three different effects could simultaneously exist to explain why individuals behave similarly in the same group (Manksi 1993). The first is the endogenous peer effect, where an individual's behavior is influenced by the behavior of her peers. The second is the exogenous or contextual peer effect, where an individual's behavior is influenced by the exogenous characteristics of her peers. The third is the correlated effect, where individuals behave similarly because they are alike or face similar environments. Among these three effects, only endogenous and exogenous peer effects represent the impact of real social interactions.

In virtual communities, peers communicate with each other through online conversations and social ties are formed via a digitally mediated platform. Online peers rely on text-based communication, which is a less rich medium than face-to-face communication (Daft and Lengel 1984). The strength of ties among peers is weaker due to lower emotional intensity, lower intimacy, and less amount of time spent on interpersonal interactions online (Granovetter 1973). Therefore, it is natural to question the degree of peer influence in a virtual environment. We conjecture that behavioral influences from peers are still present in 
virtual IOCoPs, as virtual communities "represent a vast reservoir of social and economic influence" (Bapna and Umyarov 2015, pp. 2015). Similar to offline settings, online peers share common interests in discussion topics and exchange related information and thoughts.

Endogenous peer effects suggest that peers may affect a focal individual through their actions. The reason is that individuals can observe, learn, and replicate the actions of others in the context of social interactions (Bandura 1977). Peers' actions provide an appropriate cue to encourage the individual to match these acts. An individual can also develop perceived proximity to her peers via online communication (Wilson et al. 2008), and be convinced by her peers to behave similarly. For instance, through a randomized field experiment, Bapna and Umyarov (2015) find that a user's odds of buying a product increase $50 \%$ due to her adopting peers.

Exogenous peer effects refer to the fact that peers may affect a focal individual through their characteristics, which are "thought to proxy for ability or other unobservables" (Cooley 2010, pp. 8). Research in education demonstrates that students' achievement is affected by their peers' race, sex, and socioeconomic status (Cooley 2010). Peers' characteristics influence a focal individual student via complex mechanisms, such as changing perceptions of the student or bringing additional family resources to the group. For instance, Bramoullé et al (2009) find that friends' working parents (e.g., an attribute of peers) are beneficial to a student's recreational activities, since they bring better access to those activities. In our case, peers' characteristics come from two levels, including peers' individual level attributes and organizational level attributes. Those visible characteristics can matter, as users sometimes want to identify with certain types of peers by mimicking their behavior. At the individual level, if a user's peers are more capable and pro-social, they can better engage the user in conversations and knowledge exchange. When one's peers receive more help from the IOCoP, the individual may also be motivated to participate more since she would expect the same from the community. At the organizational level, a user may be more encouraged to exchange knowledge with her peers, who come from consortium member companies. Users' confidence in and identity with the IOCoP may increase if more of their peers represent organizations promoting and supporting the standards discussed in the community. She also 
enjoys better opportunities to tap into organizational knowledge base brought by her online peers, if her peers' organizational knowledge level is higher. Overall exogenous peer effects suggest spillovers of peers' characteristics, and we hypothesize the following:

H3a: A focal individual's level of participation in a virtual IOCoP is positively related to the endogenous peer effects (i.e., level of peer participation) she receives.

H3b: A focal individual's level of participation in a virtual IOCoP is positively related to the exogenous peer effects (i.e., peer characteristics) she receives.

\subsection{Individual Level Control Variables}

Based on our prior discussion, we control individual level motivation in three categories: capability constraints, utilitarian motives, and pro-social orientation.

Tenure: Individuals' community participation is constrained by their relevant capabilities. In virtual IOCoPs, participants from different organizations exchange information and knowledge related to their professional practices (Wenger et al. 2002). In order to engage meaningfully in community activities, they need to understand the common area of expertise, and be capable of using shared language and vocabulary. It takes time for individuals to accumulate experience, master relevant expertise, and understand the norms of their specialized fields (Wasko and Faraj 2005). Thus, longer tenure helps individuals to more actively participate in virtual IOCoPs.

Informational Benefits: Individuals are motivated to participate in a community if they expect to receive rewards from doing so. Such a utilitarian perspective is adopted by social exchange theory, which suggests that self-interested individuals are interdependent in social interactions and expect to receive something back in social exchange (Blau 1964). Utilities derived from community participation include reputation enhancement, personal development, satisfaction, and intrinsic drive to acquire knowledge (Ciffolilli 2003; Peddibhotla and Subramani 2007). In our study, we focus on informational benefits obtained by individuals. Individuals can learn new knowledge from a virtual IOCoP, since such a community breaks knowledge isolation caused by organizational boarders and increases learning potential 
through diversified experiences (Moingeon et al. 2006). Individuals are more likely to be motivated to participate in a virtual IOCoP if they receive valuable information produced by the community.

Structural Capital: In addition to self-interests, individuals are also encouraged by their pro-social behavior (Wiertz and Ruyter 2007; Xia et al. 2012). For example, Wasko and Faraj (2005) study the participation and helping behavior in a virtual IOCoP and find that altruism, reciprocity, and community interest created by structural capital are important motivating factors. In this research, our dataset allows us to examine one important social variable, structural capital. People centrally located in the community have higher levels of structural capital, since they have more direct ties with other individuals. Those people tend to participate more as they "are more likely to understand and comply with group norms and expectations" (Wasko and Faraj 2005, pp. 41). More social ties with other individuals suggest more social interactions, which help individuals form habit of cooperation.

\section{Data Description}

In this section, we provide an overview of the virtual IOCoP serving as the empirical setting for our study, and describe the data that we extracted from the platform.

\subsection{Empirical Setting}

The virtual IOCoP examined here is an online discussion forum created and hosted by the FIX Trading Community, a non-profit and neutral standard body responsible for defining, managing, and promoting usage of the Financial Information eXchange (FIX) protocol as an enabler for electronic financial trading. The FIX protocol is a standardized messaging language for the automated trading of financial instruments. Software developers can create commercial or open-source software around these standards, which enables firms to transact in an electronic, transparent, cost efficient, and timely manner ${ }^{1}$. The FIX protocol is the de facto standard for pre-trade and trade communication in the global equity markets, and is expanding across the foreign exchange, fixed income, and derivative markets. It is gaining increased attention within the financial exchanges community, as "virtually every major stock exchange

${ }^{1}$ http://www.fixtradingcommunity.org/pg/structure/about/overview, last accessed on August 15th, 2016. 
and investment bank uses FIX for electronic trading, alongside the world's largest mutual funds, money managers and thousands of smaller investment firms"2 . Exchanges that have adopted the standard include NASDAQ, Irish Stock Exchange, Korea Exchange, London Stock Exchange Group, and many others. The standard is open and free, and developed by voluntary efforts from member organizations in the FIX Trading Community.

The FIX Trading Community was established in 1990's by several founding firms, including Fidelity Investments and Salomon Brothers, to promote adoption of the standard. It engages both financial institutions and IT vendors to develop the specifications. Membership dues collected by the consortium enables it to manage and expand the use of the FIX protocol. On its website, the consortium hosts discussion forums, which are open for both member and non-member organizations.

The most active and successful online discussion forum on the FIX Trading Community's website is the General Q/A forum, which is selected as the virtual IOCoP to study in this research. It has existed since 1997 and the volume of posts in the community is fairly stable during the 12-year study period. Due to its work-related nature, the community's activity level appears lower than a typical online community as only professionals that work with the FIX Protocol would exchange knowledge in the community. Although not mandatory, individuals are encouraged to disclose their own names as well as their companies' names in order to promote trust and open culture in the community ${ }^{3}$. As one individual wrote in the post that: “...Your name and company indicate that you would like to stay anonymous. There is nothing to be afraid of and you will probably get more feedback if you are willing to share your identity."

In this virtual IOCoP, information is organized by message threads. The first message in each thread is a question asked by one individual, and the following messages in the same thread are answers or further discussion contributed by other individuals. For each message, we can see the posting individuals' identification information, posting time, and the full content of each message. Figure 1 shows an example of the message threads in this community.

${ }^{2}$ http://www.fixtradingcommunity.org/pg/main/who-uses-fix/fix-version, last accessed on August 15 ${ }^{\text {th }}, 2016$.

3 Over the 12 years, only $15 \%$ of participants did not disclose any company information. 
$<<---P l e a s e$ insert Figure 1 here--- $>>$

\subsection{Variable Description}

In order to have reasonable size of observations for online messages, and consequently sufficient estimation precision, the time period or temporal unit is set as a year. The response variable in this study is users' participation level in the IOCoP, which is assessed by both the quantity and quality of participation. To accurately assess the volume of participation in a given year, we examined two measures for each individual: number of questions asked (\# of questions), and number of answers provided (\# of answers). The formal represents active knowledge consumption ${ }^{4}$, while the latter represents knowledge contribution. These are two distinct yet equally important types of behavior in virtual communities (Zheng et al. 2013).

We used helpfulness of answers contributed (Helpfulness) to quantify the quality of participation. Following Wasko and Faraj (2005), we reviewed and rated messages that answered the posted questions as very helpful (received a score of 4), helpful (received a score of 3), somewhat helpful (received a score of 2), and not helpful (received a score of 1). Helpfulness scores were averaged in a given year for each individual. If an individual did not provide any answer to others in a given year, she received a Helpfulness score of zero. We recruited two independent annotators with adequate domain knowledge to evaluate the helpfulness of all the answering messages. An inter-rater reliability check using Cohen's weighed kappa (Cohen 1968) was 0.724, indicating a substantial inter-rater agreement (Landis and Koch 1977). All rating discrepancies were jointly reviewed and reconciled by three co-authors.

The individual-level variables include tenure, informational benefits, and degree centrality. Tenure measures how long an individual has participated in the virtual IOCoP and reflects her cognitive capital (Wasko and Faraj 2005). Informational benefits reflect the average quality of answers an individual receives during a time period. High quality answers help individuals solve technical questions and learn from others. Degree centrality reflects an individual's structural capital in the network (Wasko and Faraj

\footnotetext{
${ }^{4}$ Passive knowledge consumption (i.e., message browsing behavior) cannot be captured in our dataset.
} 
2005), and is measured by the weighted number of unique individuals that a focal person is connected with in the conversation network, where the weight is the total number of posts sent and received between the focal individual and the peers. Such weights can be considered as a measure for the strength of ties.

Based on the aforementioned discussion, we include two organizational-level variables. Member is a dummy variable indicating whether an individual's organization is a member of the FIX Trading Community in a given year. It was coded based on membership records provided by the consortium management. Number of unique individuals from the same organization answering questions in the IOCoP in a given year is used as a proxy to measure organizational knowledge. The rationale is due to "the role of the individual as the primary actor in knowledge creation and the principle repository of knowledge...is essential to piercing the veil of organizational knowledge and clarifying the role of organizations in the creation and application of knowledge" (Grant 1996, pp. 121).

We also control for additional heterogeneities at three different levels. At the individual level, we control for one-timer, which indicates whether an individual only participates in the IOCoP for one time period and never comes back. One-timer is not retained by the community, and is less likely to participate actively due to lack of identity or emotional attachment to the community (Blanchard and Markus 2004). At the peer level, we control aggregated peer contribution from the entire community, measured by the total number of posts in the virtual IOCoP in a year. It reflects social norms formed among all community participants. At the organizational level, we control for different organizational affiliations. Our IOCoP has three types of individuals: the first type comes from IT vendors who develop standards-compliant systems; the second type works for software user organizations who adopt the standards for the automated trading of financial instruments with other financial institutions. IT vendors and user organizations represent the supply and demand sides of the standards and have different motivations to develop and use the standards (Zhao et al. 2011b). The third type consists of self-represented individuals, who either claim themselves as self-employed or do not disclose employer information in the IOCoP. Two dummy variables, IT vendor ( 1 indicating the organization is an IT vendor; 0 otherwise) and 
software user ( 1 indicating the organization is a software user of the FIX protocol; 0 otherwise), are used to differentiate three types of organizational affiliations.

Table 1 shows the variable description and the summarized descriptive statistics. Although messages posted in the IOCoP can be traced back to 1997 , we only started our data collection from 2001 . The primary purpose is to ensure the variation in the key constructs (e.g., information benefits, member, and organizational knowledge) in the years of data. The variation in early years' data is low and may lead to the model estimation problem. Overall, 1803 unique individuals from 957 organizations have posted 6698 messages over the 12 years.

$<<---P l e a s e$ insert Table 1 here--->>

\subsection{Network Construction}

Considering the longitudinal nature of our dataset, we construct a social network for each year in the virtual IOCoP. In the network, each node representing a user of the virtual IOCoP posting and/or answering questions online. The connections of social networks can be defined by varies of relationships and interactions, such as social ties, (Wuchty, 2009), communication, (Godes and Mayzlin, 2004), and network weighting mechanism, (Burt, 1987). As peer influence can happen via both social relation and behaviors, we use communication in an IOCoP to define the connection. The arcs, which are the directed connections between nodes, represent the reply-to or answer interactions among individuals. When defining the arcs of a network, we consider all the questions and answers created in a full calendar year. If individual $i$ posts a question and individual $j$ answers $i$ 's question, then an $\operatorname{arc}$ from $j$ to $i$ is defined. An illustration about how the connections (arc) of the network are defined is provided in Figure 2 below. Based on the arcs defined, an adjacency matrix $\mathbf{A}_{t}$ represents the network structure in year $t . \mathbf{A}_{t}$ is time varying as participants are changing in the virtual IOCoP from year to year. In each adjacency matrix $\mathbf{A}_{t}$, the value for element is binary. $A_{j i t}=1$ if individual $j$ answers questions by $i$ in year $t$, and 0 otherwise. Since the network is directed, the adjacency matrix is asymmetric, and $A_{j i t}=1$ does not necessary mean $A_{i j t}=1$, unless $i$ answeres question from $j$ too. Since an individual cannot answer her own question, the diagonals of all the adjacency matrices $\mathbf{A}_{t}$ are set as zeros. 
$<<---P l e a s e$ insert Figure 2 here--->>

Heterogeneity in the question asking and answering behavior among all the IOCoP users can be high. Some individuals may be more active in the IOCoP, post more questions or more interesting questions, and attract many other users' answers, resulting in many neighbors. Other individuals may not have many questions or not attract many answers, resulting in fewer neighbors. For individuals with many neighbors, the influence from each neighbor is not at the same size as that of individuals with very few neighbors. To avoid the inflation of peer effects from a large number of neighbors, we normalize the elements of $\mathbf{A}_{t}$ column-wise,

$$
\mathbf{W}_{t}=\left\{W_{j i t}\right\}=\left\{\frac{A_{j i t}}{n_{i t}}\right\}
$$

where $\mathbf{W}_{t}$ is the resulted normalized adjacency matrix, $n_{i t}$ is the total number of neighbors for individual $i$ in year $t$

\section{Econometric Analysis}

In this section, we define the econometric model in order to identify both exogenous and endogenous peer effects. The methodology to empirically identify, disentangle, and estimate both endogenous peer effects and exogenous peer effects is a recent development (Bramoullé et al 2009; Pool et al. 2014). Studies that scrutinize the joint effect of personal attributes and peer effects often do so through the use of network autocorrelation models (Valente 2005), since individuals' behavior is interdependent. Based on spatial econometrics, Bramoullé et al. (2009) propose an extension of the linear-in-means model to empirically identify endogenous and exogenous peer effects. The prerequisite is that social interactions among individuals are observable, and a directed social network can be constructed and factored into econometric estimations. In addition, correlated effects are assumed to be fixed within self-contained networks. Bramoullé et al.'s approach is general enough to distinguish peer effects in many networks. It has been applied in social networks where ties are defined by using face-to-face interactions, such as recreational service choices in US high schools and middle schools (Bramoullé et al 2009), college major 
decisions in Bocconi (De Giorgi et al. 2010), and academic achievement in primary schools in Uruguay (De Melo 2011). In those offline networks, peer effects have been shown positive and significant, indicating that individuals' choices are affected by their peers' behavior and exogenous characteristics.

However, the phenomenon we study presents two technical challenges. First, the individual attributes collected and the networks in which these individuals are embedded are longitudinal. Second, there are possible unobserved individual level factors coming from the virtual platform correlating with the observed attributes. To address these issues, we extend Bramoullé et al (2009) by adding a network autocorrelation component to accommodate both panel data and fixed effects. This extension allows us to investigate the peer influence in virtual communities.

\subsection{Model}

We extend Bramoullé et al.'s model (2009) to study individuals' virtual IOCoP participation decision, when such decision might be associated with both own characteristics and peer effects in the network. Notations of the model are provided in Table 2.

The structural model for a focal virtual IOCoP individual $i$ in year $t$ is characterized as:

$$
y_{i t}=\beta \frac{\sum_{j \in P_{i t}} y_{j t}}{n_{i t}}+\gamma x_{i t}+\delta \frac{\sum_{j \in P_{i t}} x_{j t}}{n_{i t}}+\zeta \frac{\sum_{j \in P_{i t}} H_{j t}}{n_{i t}}+\eta_{i}+\varepsilon_{i t}, \mathrm{E}\left(\varepsilon_{\mathrm{it}} \mid x_{i t}=0\right)
$$

where $y_{i t}$ is the decision made by individual $i$ in year $t . X_{i t}$ is a $1 \times K$ vector of individual observable attributes, including both individual-level attributes and organizational features associated with individual $i$. Each individual $i$ has a peer group $P_{i t}$ who has conversations in the virtual IOCoP at time $t$, with a size of $n_{i t} . \beta$ captures the endogenous peer effect from the network, and $\delta$ captures the exogenous peer effect. Since we have a panel dataset describing individuals' attributes and decisions in different years, we also include fixed effects $\eta_{i}$ to model the correlated effect, which refers to individuals in the same communication network tend to have same participation behavior because of similar unobserved attributes. Having such term in the model can account for unobserved attributes common to each individual in the network of the same time period. This term can also allow for correlations between the 
unobserved attributes of individuals, e.g. years of experience in the industry, and observed individual attributes in $\mathbf{X}_{t}$, e.g. years of IOCoP participation.

$H_{i t}$ is a $1 \times L$ vector of measures representing different dimensions of homophily for individual $i$ in time period $t$. According to the literature, homophily is associated with both demographic attributes, such as age and gender (McPherson et al., 2001), and attributes that affect how individuals behave similarly (Lazarsfeld and Merton 1954). Since the outcomes we want to study are online behaviors, such behaviors are more likely to be associated with users' homophily in platform usage pattern. Following Aral et al. (2009), we used observed attributes representing multiple IOCoP access patterns to measure homophily, such as post time (i.e., frequently post time) and social circle (i.e., number of unique users communicated with). We explicitly include the term $H_{i t}$ in order to control for effects of homophily that may come from neighbors of each individual in the social network.

The model in matrix notation can be described as:

$$
\begin{aligned}
\mathbf{y}_{t}=\beta \mathbf{W}_{t} \mathbf{y}_{t}+ & \boldsymbol{\gamma} \mathbf{X}_{t}+\boldsymbol{\delta} \mathbf{W}_{t} \mathbf{X}_{t}+\zeta \mathbf{W}_{t} \mathbf{H}_{t}+\eta+\boldsymbol{\varepsilon}_{t} \\
& \mathrm{E}\left(\boldsymbol{\varepsilon}_{t} \mid \mathbf{X}_{t}, \mathbf{H}_{t}, \mathbf{W}_{t}\right)=0 \\
& \mathbf{X}_{t}=\boldsymbol{\mu}+\mathbf{e}_{t} \\
& \left(\boldsymbol{\eta}, \mathbf{e}_{t}\right) \sim \operatorname{MVN}(\mathbf{0}, \mathbf{\Omega}) \\
& \mathbf{\Omega} \sim \operatorname{InvWishart}\left(\boldsymbol{\Sigma}_{\Omega}, d_{\Omega}\right)
\end{aligned}
$$

where $\mathbf{y}_{\mathrm{t}}$ is an $n \times 1$ vector of response variables in each time period.

$$
<<--- \text { Please insert Table } 2 \text { here--- }>>
$$

In this study we investigate three online behaviors about individuals, number of questions asked, number of answers provided, and average helpfulness of answers by individual $i$ in year $t$. The specifications of these different response variables are shown below. The definition of response variables $\mathbf{y}_{t_{-} Q U E S,}, \mathbf{y}_{\_} \_A N S$, and $\mathbf{y}_{t_{-} H E L P}$ is provided in Table 2.

$$
\begin{aligned}
& \mathbf{y}_{t_{-} Q U E S}=\beta \mathbf{W}_{t} \mathbf{y}_{t_{-} Q U E S}+\gamma \mathbf{X}_{t}+\delta \mathbf{W}_{t} \mathbf{X}_{t}+\zeta \mathbf{W}_{t} \mathbf{H}_{t}+\eta+\boldsymbol{\varepsilon}_{t} \\
& \mathbf{y}_{t_{-} A N S}=\beta \mathbf{W}_{t} \mathbf{y}_{t_{-} A N S}+\gamma \mathbf{X}_{t}+\delta \mathbf{W}_{t} \mathbf{X}_{t}+\zeta \mathbf{W}_{t} \mathbf{H}_{t}+\eta+\boldsymbol{\varepsilon}_{t} \\
& \mathbf{y}_{t_{-} H E L P}=\beta \mathbf{W}_{t} \mathbf{y}_{t_{-} H E L P}+\gamma \mathbf{X}_{t}+\delta \mathbf{W}_{t} \mathbf{X}_{t}+\zeta \mathbf{W}_{t} \mathbf{H}_{t}+\eta+\boldsymbol{\varepsilon}_{t}
\end{aligned}
$$


$\mathbf{W}_{t}$ is an $n \times n$ matrix describing the post conversations between individuals in the IOCoP, with

$W_{j i t}=\frac{1}{n_{i t}}$ if individual $j$ responds to individual $i$ 's original post in year $t$, and 0 otherwise. $n_{i t}$ is the total number of individuals who respond to $i$ in year $t$. Since $\mathbf{W}_{t}$ describes directed relationships between individuals, it is an asymmetric matrix. $\boldsymbol{\eta}$ is an $n \times 1$ vector of the individual level fixed effects for all $n$ participants. Covariates $\mathbf{X}_{t}$ can be represented as mean $\boldsymbol{\mu}$ with time-variant error $\mathbf{e}_{t \cdot} \boldsymbol{\eta}$ and $\mathbf{e}_{t}$ follow a multivariate normal distribution with parameters $\mathbf{0}$ and $\boldsymbol{\Omega}$. $\mathbf{\Omega}$ follows an inverse Wishart distribution with parameters $\boldsymbol{\Sigma}_{\Omega}$ and $d_{\Omega}$. $\mathbf{H}_{t}$ is the matrix of attributes representing different dimensions of homophily in period $t$. We include two time variant dimensions representing individuals homophily in online usage pattern, post time and social circle.

\subsection{Identification Strategy}

The "reflection problem" (Manski 1993) can be considered as a major challenge in identification of peer influence. It refers to the endogeneity of, or the inability of separating the endogenous effect, the peer influence, from the exogenous effect. Bramoullé et al. (2009) proposed a spatial autoregressive model that can achieve the identification. They showed that when a network contains an individual's three-hop or higher neighbors, peer influence can be identified. Bramoullé et al.'s principle is that the behavior of a neighbor's neighbor can be used as an instrumental variable, because it is not a determinant of response variable $\mathbf{y}$, despite still being correlated with individual attributes. Considering a simplified structural model yet still containing all necessary terms about $\mathbf{y}_{t}$ below. The model only contains one predictor and one variable measuring homophily, thus $\mathbf{x}_{t}$ and $\mathbf{h}_{t}$ are both vectors at $n \times 1$.

$$
\mathbf{y}_{t}=\beta \mathbf{W}_{t} \mathbf{y}_{t}+\gamma \mathbf{x}_{t}+\delta \mathbf{W}_{t} \mathbf{x}_{t}+\zeta \mathbf{W}_{t} \mathbf{h}_{t}+\boldsymbol{\eta}+\boldsymbol{\varepsilon}_{t}
$$

The identification of peer influence is achieved if and only if all the parameters in the structural model, $\alpha, \beta, \gamma$, and $\delta$, can be uniquely recovered from the reduced form below.

$$
\begin{aligned}
& \left(I-\beta \mathbf{W}_{t}\right) \mathbf{y}_{t}=\gamma \mathbf{x}_{t}+\delta \mathbf{W}_{t} \mathbf{x}_{t}+\zeta \mathbf{W}_{t} \mathbf{h}_{t}+\boldsymbol{\eta}+\boldsymbol{\varepsilon}_{t} \\
& \mathbf{y}_{t}=\left(I-\beta \mathbf{W}_{t}\right)^{-1}\left(\gamma+\delta \mathbf{W}_{t}\right) \mathbf{x}_{t}+\left(I-\beta \mathbf{W}_{t}\right)^{-1} \zeta \mathbf{W}_{t} \mathbf{h}_{t}+\left(I-\beta \mathbf{W}_{t}\right)^{-1}\left(\boldsymbol{\eta}+\boldsymbol{\varepsilon}_{t}\right)
\end{aligned}
$$


The (model above) can be interpreted as instrumental variables (IVs). From the series expansion of

the model: $(I-\mathbf{A})^{-1}=\sum_{k=0}^{\infty} \mathbf{A}^{k}$, where $\mathbf{A}=\beta \mathbf{W}_{t}$

A within network transformation (demean) on $\mathbf{y}_{t}$ is given by:

$\left(I-\mathbf{W}_{t}\right) \mathbf{y}_{t}=\beta\left(I-\mathbf{W}_{t}\right) \mathbf{W}_{t} \mathbf{y}_{t}+\left(I-\mathbf{W}_{t}\right)\left(\gamma+\delta \mathbf{W}_{t}\right) \mathbf{x}_{t}+\zeta\left(I-\mathbf{W}_{t}\right) \mathbf{W}_{t} \mathbf{h}_{t}+\left(I-\mathbf{W}_{t}\right)\left(\boldsymbol{\eta}+\boldsymbol{\varepsilon}_{t}\right)$

Its reduced form is given by:

$\left(I-\mathbf{W}_{t}\right) \mathbf{y}_{t}=\left(I-\beta \mathbf{W}_{t}\right)^{-1}\left(\gamma+\delta \mathbf{W}_{t}\right)\left(I-\mathbf{W}_{t}\right) \mathbf{x}_{t}+\left(I-\beta \mathbf{W}_{t}\right)^{-1}\left(I-\mathbf{W}_{t}\right) \zeta \mathbf{W}_{t} \mathbf{h}_{t}+\left(I-\beta \mathbf{W}_{t}\right)^{-1}\left(I-\mathbf{W}_{t}\right)\left(\boldsymbol{\eta}+\boldsymbol{\varepsilon}_{t}\right)$

Expected peer influence in the network, conditional on $\mathbf{x}_{t}$ and homophily is: $\mathrm{E}\left(\left(I-\mathbf{W}_{t}\right) \mathbf{W}_{t} \mathbf{y}_{t} \mid\left[\mathbf{x}_{t} \mathbf{h}_{t}\right]\right)$, where $\left[\mathbf{x}_{t} \mathbf{h}_{t}\right]$ is the augmentation of matrices $\mathbf{x}_{t}$ and $\mathbf{h}_{t}$. Using Bramoulle et al.'s proposition (2009), variables $\left[\left(I-\mathbf{W}_{t}\right) \mathbf{W}_{t}^{2}\left[\mathbf{x}_{t} \mathbf{h}_{t}\right],\left[\left(I-\mathbf{W}_{t}\right) \mathbf{W}_{t}^{3}\left[\mathbf{x}_{t} \mathbf{h}_{t}\right], \ldots.\right]\right.$ can be used as valid IVs for $\left(I-\mathbf{W}_{t}\right) \mathbf{W}_{t} \mathbf{y}_{t}$. This condition is equivalent to $I, \mathbf{W}_{t}, \mathbf{W}_{t}^{2}$, and $\mathbf{W}_{t}^{3}$ are linearly independent, which ultimately can be converted to the condition of $\mathbf{W}_{t}$, s diameter is greater than or equal to 3 . Figure 3 below shows an example of an extracted subnetwork in one year. A directed edge from node $i$ to node $j$ represents that individual $i$ responds to $j$ 's post in a particual period. From the topology of the network, we can easily find that there are paths between nodes with distance greater than or equal to three. The linear independence among matrices representing peer influence $\boldsymbol{I}, \mathbf{W}_{t}, \mathbf{W}_{t}{ }^{2}$, and $\mathbf{W}_{t}{ }^{3}$ is also quantitatively confirmed for the network in all time periods. Thus the identification condition by Bramoullé et al.' (2009) is satisfied. An illustration of the identification condition is shown in Online Appendix.

$<<---P l e a s e$ insert Figure 3 here--->>

\section{Results}

In this section, we present the results of our main model and those of some alternative model specifications.

\subsection{Main Results}

The results of our main model are presented in Table 3, which reports the coefficients of own characteristics, endogenous peer effects, and exogenous peer effects. Within the own characteristics and 
exogenous peer characteristics, we cluster the results into two levels, individual level and organizational level. Exogenous peer effects assess "the influence of exogenous peer characteristics" (Bramoullé et al. 2009; pp. 41), and the set of exogenous peer characteristics corresponds exactly to one's own characteristics. According to the identification conditions specified by Bramoullé et al. (2009) and the 2SLS procedure (Lee 2003), we are able to obtain asymptotically optimal estimates of the peer effects.

$$
<<--- \text { Please insert Table } 3 \text { here--->> }
$$

Organizational factors have significant influences on individuals' participation in the virtual IOCoP. Individuals from a member organization ask fewer questions and provide more answers. The answers provided by such participants are shown to be more helpful, indicating participants from member organizations play a positive role in the knowledge contribution to the forum, both in terms of quality and quantity. H1 is supported in terms of quality and quantity of the knowledge contribution. We found participants whose organizations possess richer internal resources and institutional knowledge tend to ask less and answer less, while the answers provided are on average more helpful. This finding suggests that participants from such organizations have a lower incentive to seek and share knowledge externally; however, the knowledge contributed is of higher quality. H2 is supported in terms of quality of the knowledge contribution. Individuals from vendor organizations exhibit a similar pattern to those from member organizations. Individuals from software user organizations show an opposite pattern - they tend to answer less and the quality of their answers is lower. This clear contrast in behavior indicates the differential influences of their organizational factors.

Endogenous peer effects are significant and positive in all three model estimations, suggesting that peers' participation behavior has a positive impact on the participation of the focal individual, in terms of both quantity, as measured by the number of questions and answers, and quality, as measured by helpfulness of the answers. Thus, H3a is supported in all dimensions of participation.

Exogenous peer effects are, at large, influential in the three models. Depending on the aspects of the participation, however, the significance of the impacts varies among different exogenous peer characteristics. $\mathrm{H} 3 \mathrm{~b}$ is partially supported in each of the three dimensions of participation. In terms of the 
number of questions asked, the results show that one tends to ask more questions if the peers are more senior, have more organizational knowledge, or are from a member organization. This implies that individuals are more actively seeking knowledge in a community with more senior users, more member users, or people who possess more institutional knowledge. With regard to the number of questions answered, one tends to give more answers if peers are more senior, more centrally located in the network, or has an organizational affiliation, suggesting that individuals are more actively contributing knowledge to a community with more senior users, users with higher structural capitals, or more participants who are affiliated with organizations. For helpfulness, the results show that an individual provides more helpful answers if peers are more senior, more centrally located, belong to a member organization or a vendor. Additionally, an individual's own posts tend to be less helpful when peers have received more information benefits, indicating the existence of diffusion of responsibility in such a community.

Overall, peer effects play an important role in shaping participation in virtual IOCoPs, indicating that social ties are influential in such communities. Compared to endogenous peer effects, exogenous peer effects vary based on the dimensions of the participation. In addition, coefficients of exogenous peer effects are in general smaller than those of endogenous peer effects. These findings suggest that one's participation behavior is more influenced by peers' actions than by peers' characteristics.

The coefficients of the control variables at the individual level are mostly significant and conforming to existing literature. Particularly, individuals who receive more information benefits tend to ask more questions and contribute higher quality answers, indicating a reciprocity behavior. Tenure and degree centrality have positive and significant impacts on participation. Individuals who stay in the community for a long time tend to ask less while answering more and contributing higher quality answers. Individuals who are more central in the network tend to ask more, answer more and provide more helpful answers. Surprisingly, we find that one-timers in their occurred single period participate more actively in the IOCoP than their retained counterparts. One possible explanation is that those one-timers might have a greater need of knowledge sharing in that single period. One-timers have no intention of long-term stay, and their participation is mainly driven by the urge to ask/answer specific questions. While retained users' 
knowledge exchange activities span over years, one-timers concentrate their activities in just a single time period. Moreover, although not socially identified with the community, one-timers might bring different perspectives and new insights, eliciting more online discussions.

\subsection{Robustness Checks}

\subsubsection{Propensity Score Matching}

To further infer causality between the endogenous peer influences and IOCoP contribution, we employed DID matching strategies to explore the robustness of the estimation of our model. The DID matching estimator compares the change of the post decision of individuals from the beginning of time period to the end of time period for each period when controlling for peer influence. We constructed one control group and one treatment group, out of the population data (Table 4). The control group consists of individuals that are isolated from the IOCoP post conversation network (represented as Type 1 structure) from the beginning of each time period until the end of each time period, meaning that users in the control group are not affected by peer influence. The treatment group consists of users who are isolated from the post conversation network at the beginning of each time period but by the end of the time period become a pendant node - connecting to exactly one user from the network (represented as Type 2 structure in Table 4). Individuals in the treatment group are only affected by peer influence from their one and only neighbor who are content creators. We focus on the structure types as the event of interest. Following the structure type change, the posting decision of users in the treatment group are compared with those of matching users in the control group. The impact of endogenous peer influence is inferred by comparing the difference between the participation change in the treatment group and the control group. The matching parameters include attributes representing homophily, such as working industry, post time and social circle, etc. Also, degree centrality is added in the matching variables in order to control for the peer influence of non-creators. We employed Propensity Score Matching (PSM) (Leuven and Sianesi 2003) for average treatment effects estimation. Finally, PSM is run on all time periods. The results are shown in Tables 4 and 5 .

$<<---P l e a s e$ insert Table 4 here--- >> 
$<<---$ Please insert Table 5 here--->>

As we expected, the DID matching results indicate that the peer influences will significantly affect the IOCoP participation decision, in all three measures, asking, answering, and helpfulness. For asking question, when an individual is affected by peer influence from one neighbor, she provide 0.16 more answers $(\mathrm{p}<0.01)$ than the matched player from the control group. For questions answering, a participant answers 0.27 more posts $(\mathrm{p}<0.01)$ than the matched sample from the control group who does not receive any peer influence. The helpfulness of the answer provided by a participant receives peer influence from one neighbor also is 0.24 higher than the control group. The PSM coefficients suggest that in network, peer influence leads to higher number of asking, answering and helpfulness. All results confirm what we find in the main model analysis.

\subsubsection{Alternative Model Specifications}

We explored alternative specifications for the main model to test the robustness of our results. Table 6 reports the estimation results of the three models excluding exogenous peer effects. The rationale for this specification is that, while peers' actions are observable in virtual communities, their contextual factors that correspond to the exogenous peer effects, such as organizational knowledge, may be less obvious to other members, therefore having less impact on individuals' participation behavior. As a robustness check, we run a separate set of models explicitly excluding exogenous peer effects. As shown in Table 6, the results remain consistent with those in the main model. The effects of organizational factors and endogenous peer effects remain significant.

$<<---P l e a s e$ insert Table 6 here--->>

Additionally, we experiment with three alternative model specifications. The first experiment deals with the definition of membership. Instead of using a binary variable "Member" to indicate whether one's organization is a member of the FIX Trading Community in a given year as in the main model, we use membership tenure to measure the membership status. Membership tenure is defined as the number of years an organization has been a member of the standard body. The assumption is that, the tenure status of an organization as a member in the community may have different influences on its employees' 
participation behavior. The more senior an organization is as a member, the more communal activities we expect to observe from its employees. The second experiment deals with the measure of the organizational knowledge. While in the main model we use the total number of individuals from an organization responding to questions in a given year as the measure for organizational knowledge, in the alternative model, we use the accumulative number of active individuals from an organization responding to questions up to a given year. The rationale is that, the organizational knowledge usually can be carried over time. The knowledge shared in previous years may be retained within an organization and adds to the overall body of knowledge of an organization. The third experiment deals with the measure of an individual's structural capital. Rather than degree centrality, in the alternative specification, we use betweenness centrality of an individual to measure one's structural capital. Betweenness centrality is defined as the number of shortest paths that passes through a focal node in a network; it is an alternative measure of a node's centrality (Freeman 1977). In our setting, betweenness is computed as the number of pairs that are connected through the focal individual in the conversation network. Only the shortest path between pairs is considered when calculating the betweenness centrality. Overall, the results from the three sets of experiments are very similar to those reported in our main model, and our results are robust to all the alternative specifications we have examined.

$<<---$ Please insert Table 6 here--- $>>$

\section{Discussion}

Virtual IOCoPs provide individuals and organizations a digital platform to exchange and co-create domain-specific knowledge across organizational boundaries. With our initial attempt to disentangle motivating factors across levels, we offer a deeper understanding of dynamics in these communities, which is critical to sustain collective learning and knowledge sharing. Taken together, in virtual IOCoPs whose contents are built from individual initiatives, contextual motivating factors are influential in determining individuals' community participation. Individuals are not only self-driven, but also subject to peer effects within and organizational influences outside the community. Our study differentiates knowledge consumption from knowledge contribution and assesses participation behavior both 
quantitatively and qualitatively. Our research model can be generalized to any virtual community setting where multi-level participation incentives exist.

\subsection{Peer Spillovers in the Virtual IOCoP}

The economic and sociology literature has long recognized the phenomenon of peer spillovers in offline social networks, where individuals' preferences, expectations, and constraints can be shaped and affected in the process of interacting with their peers (Jackson 2006; Manski 2000). To investigate whether peer effects exist in the virtual environment, we apply a novel identification strategy in our dataset. We find evidence of both endogenous and exogenous peer effects, complementing prior findings in the offline environment (De Giorgi et al. 2010; De Melo 2011; Lin et al. 2010).

Our results suggest that peer effects matter even in the presence of electronic weak ties formed via virtual social interactions (Constant et al. 1996). According to Granovetter's seminal paper (1973, pp. 1361), "the strength of a tie is a (probably linear) combination of the amount of time, the emotional intensity, the intimacy (mutual confiding), and the reciprocal services with characterize the tie". In our case, peers are geographically dispersed, may not know and interact with each other outside the virtual IOCoP, and focus their discussion topics on standard specifications, a non-personal subject matter. Consequently, the interpersonal ties between them are weak. However, electronic weak ties do not prevent individuals from influencing others to behave similarly. In addition, individuals are affected by certain exogenous characteristics of their online peers. By differentiating two types of peer effects, we find that endogenous peer effects are in general more influential than exogenous peer effects in our virtual IOCoP. It suggests that individuals have a stronger tendency to do what everyone else is doing, than to adjust their behavior based on who their peers are. It is consistent with prior literature of herd behavior in various offline or online settings (Banerjee 1992; Duan et al. 2008). Individuals usually imitate their peers' actions, which generate positive network externalities and establish norms in the online community.

Most peer effects are positive with only one exception: peers' degree centrality negatively affects a focal individual's quality of knowledge contribution. One possible explanation is that diffusion of 
responsibility could happen (Leary and Forsyth 1987). When an individual is connected with peers that are centrally embedded in the social network, she might feel that those peers can get help from their other friends. Consequently, the focal individual may feel less responsible for providing high quality answers.

\subsection{Influences from the Organizations}

At the organizational level, we find that organizational contexts are important, which can simultaneously encourage as well as hinder individuals' participation in virtual IOCoPs. It is well known that organizations can influence their employees' participation in virtual communities through monetary incentives (Roberts et al. 2006). Our results further demonstrate that even without strategic involvement, organizations can still shape their employees' behavior in virtual IOCoPs where participation is autonomous and self-determined.

Our findings highlight the importance of attributes that can signal organizations' interests and commitment to professional knowledge exchanged in IOCoPs. Specially, we explore the role of organizational consortium membership status in our research setting. Individuals from member organizations of the FIX Trading Community ask fewer questions and provide more and better answers than those from non-member organizations. As a consortium-based standard body, the FIX Trading Community relies on private resource provision from member organizations to develop the open standards (Markus et al. 2006; Zhao et al. 2011b). Thus, being a member of the FIX Trading Community shows an organization's support to the technical specifications developed and promoted by the consortium. Member organizations also have greater influence in the standard consortium, since they can decide how standards should evolve by participating in the consortium committees and working groups and exercising their voting rights (Zhao et al. 2011a). By observing their organizations' membership status, individuals tend to share their organizations' beliefs in the standards and recognize their organizations' influence in the consortium. Consequently, it is easier for these individuals to build community identity in the virtual IOCoP initiated and hosted by the consortium, leading to more active participation. 
Other than the membership status, organizational affiliations also affect individuals' participation in the virtual IOCoP. IT vendors are expected to prove their technical capabilities and improve their visibility in the marketplace in order to attract more customers to use their systems or services. We find that employees from IT vendors ask fewer questions and provide more and higher quality answers. This suggests that individuals' behavior is aligned with their organizations' interests.

We find an unexpected yet interesting result at the organizational level with regard to organizational knowledge. If an individual's organization has more institutional knowledge, they create relatively less content, but of higher quality. Volume of content creation is important for a virtual IOCoP since it ensures the necessary social interaction frequency required to build shared emotional connections in the community (Blanchard and Markus 2004). Reduced volume from individuals in certain organizations suggests constraints presented by their organizational context (Peters et al. 1985). The constraints could be attributed to complementarity or competition between the work environment and the virtual community. Virtual IOCoPs are a complementary channel for individuals to access knowledge that is not available within their organizations. They also provide individuals a complementary professional community in addition to the ones naturally formed within their organizations (Nonaka 1994; Constant et al. 1996). Individuals communicate less in the virtual IOCoP when they can easily interact with their colleagues working within their organizations. However, they are able to post higher quality answers, since organizational knowledge can be transferred to individuals and empower them to do so (Bhatt 2002; Nonoka 1994).

\subsection{Implications for Research and Practice}

We contribute to the literature in several important ways. First, this study is among the first to investigate virtual IOCoPs by disentangling motivating factors of individual participation across three different levels: self, peers, and organizations. A virtual IOCoP depends on individual participants from different organizations, and focuses on work-related knowledge exchanged via digitalized social interactions. Thus, self-driven, peer effects, and organizational influence could simultaneously exist. It is critical to differentiate motivational factors across levels in order to understand various mechanisms 
community organizers can use to encourage collective learning. Second, we are the first to analyze peer effects from both endogenous and exogenous dimensions in the virtual environment, and demonstrate their differing impacts on individual participation in virtual IOCoPs. Third, we reveal nuances of organizational influence faced by individuals working in the virtual IOCoP. Organizational influences are internalized by individuals. Moreover, it is important to realize that the work environment and the virtual IOCoP are substitutes for individuals' time, effort and commitment. More knowledge available at work discourages individuals' volume of participation in virtual IOCoPs. Fourth, we confirm prior studies about the importance of individual-level incentives and suggest a strong micro foundation of knowledge management in the virtual IOCoP. Individuals' role in learning and knowledge creation is well recognized within an organization (Grant 1996; Simon 1991), and our study confirms that such a role can also be found in the inter-organizational setting. Last but not least, studies of professional communities in the financial trading sector are limited. Our research deepens the understanding of diverse forms of virtual communities, especially IOCoPs in an important industry sector.

Our findings provide community founders several important insights into better design and management of virtual IOCoPs. Due to peer spillovers, retaining active contributors in the virtual community is crucial. Those individuals are valuable not only for their own contributions, but also for how their contributions encourage their peers to do the same. Our results draw attention to the important role of long-time community participants. Individuals tend to contribute more knowledge if they have been in the virtual IOCoP for a longer time. Moreover, their seniority is observable to other online peers, who are motivated to participate more actively in the community. Attracting and retaining individuals from member organizations of the standard consortium is beneficial to our IOCoP, as member organizations are also the backbone organizations in the virtual world. Our research also demonstrates the importance of quantifying individuals' participation level in virtual IOCoPs from multiple perspectives. Knowledge consumption and provision are indeed two different types of behavior. Factors hindering knowledge consumption, such as tenure and organizational membership status, can motivate knowledge provision. Knowledge contribution quantity also differs from knowledge contribution quality. For 
instance, while organizational knowledge limits an individual's volume of participation, it encourages higher quality answers.

From an organization's standpoint, virtual IOCoPs are a complement to the formal divisionalization of expertise within it. Organizations can enhance organizational learning and innovation capabilities by allowing individuals to participate in these communities. Organizations may leverage this digital platform to gain competitive advantages and increase marketability by actively encouraging employees to use virtual IOCoPs as an alternative learning channel to access external knowledge and expertise.

\subsection{Limitations and Future Research}

As an initial attempt to develop and validate a multilevel model of virtual IOCoPs, this study has several limitations that, in turn, offer opportunities for future research. Data available through the virtual IOCoP and public sources are limited. For example, since we deal with many private firms (e.g., many specialized IT vendors), additional organizational data, such as size and financial performance, is not publicly available. Also, we can only observe active knowledge consumption and contribution based on posted messages. However, we cannot identify individuals' browsing behavior, which represent their passive knowledge consumption in the community. Although our study yields interesting findings about virtual IOCoPs, our focus on a single IOCoP in the financial security sector raises concern about the generalizability of the findings. For instance, several organizational attributes, such as consortium membership and user/vendor differences, are unique to our research setting. We propose that our results can be generalized to other industries, since the inter-organizational setting and digitalized knowledge sharing platform are widely used. Additional attributes that can signal organizations' interests and support to virtual communities should be further identified and explored.

\section{References}

Aral, S. (2011). Identifying social influence: A comment on opinion leadership and social contagion in new product diffusion. Marketing Science. 20: 217-223.

Ardichvili, A., Page, V., Wentling, T. (2003). Motivation and barriers to participation in virtual knowledge-sharing communities of practice. Journal of Knowledge Management. 7(1): 64-77.

Ahn DY, Duan JA, Mela C (2014). Managing user generated content: A dynamic rational expectations equilibrium approach. Working paper, Duke University, Durham, NC. 
Bandura, A. (1977). Self-efficacy: Towards a unifying theory of behavioral change. Psychological Review. 84(2): 191-215.

Banerjee, A. (1992). A simple model of herd behavior. Quarterly Journal of Economics. 107: 797-817.

Bapna, R. and Umyarov, A. (2015). Do your online friends make you pay? A randomized field experiment on peer influence in online social networks. Management Science. 61(8): 1902-1920.

Battersby, S.L., Verdi, B. (2015) The culture of professional learning communities and connections to improve teacher efficacy and support student learning. Arts Education Policy Review. 116(1): 2229.

Bayus, B.L. (2013). Crowdsourcing New Product Ideas over Time: An Analysis of the Dell IdeaStorm Community. Management Science. 59(1): 226-244.

Bhatt, D. (2002). Management strategies for individual knowledge and organizational knowledge. Journal of Knowledge Management. 6(1): 31-39.

Bikhchandani, S., Hirshleifer, D., Welch, I. (1992). A theory of fads, fashion, custom, and cultural change as informational cascades. Journal of Political Economy. 100(5): 992-1026.

Blanchard, A.L., Markus, M.L. (2004). The experienced "sense" of a virtual community: Characteristics and processes. The Data Base for Advances in Information Systems. 35(1): 65-79.

Blau, P.M. (1964). Exchange and Power in Social Life. Wiley, New York.

Bloor, G., Dawson, P. (1994). Understanding professional culture in organizational context. Organization Studies. 15(2): 275-295.

Brown, J., Duguid, P. (1991). Organizational learning and toward a unified view of working, learning, and innovation. Organization Science. 2: 40-56.

Bramoullé, Y., Djebbari, H., Fortin, B. (2009). Identification of peer effects through social networks. Journal of Econometrics. 150: 41-55.

Bramoullé, Y., Kranton, R., D'amours, M. (2014). Strategic interaction and networks. The American Economic Review. 104(3): 898-930.

Burt, R. S. (1987). Social contagion and innovation: Cohesion versus structural equivalence. American Journal of Sociology, 92(6):1287.

Cheung, C.M.K., Lee, M.K.O., Lee, Z.W.Y. (2013). Understanding the continuance intention of knowledge sharing in online communities of practice through the post-knowledge-sharing evaluation. Journal of the American Society for Information Science and Technology. 64(7): 13571374.

Ciffolilli, A. (2003). Phantom authority, self-selective recruitment and retention of members in virtual communities: The case ofWikipedia. First Monday, 8(12).

Cohen, J.A. (1968). Weighed kappa: Nominal scale agreement with provision for scaled disagreement or partial credit. Psychological Bulletin 70 (4): 213-220.

Constant, D., Sproull, L., Kieslet, S. (1996). The kindness of strangers: The usefulness of electronic week ties for technical advice. Organization Science. 7(2): 119-135.

Cooley, J. (2010). Desegregation and the achievement gap: Do diverse peers help? Working Paper, University of Wisconsin-Madison.

Daft, R.L., Lengel, R.H. (1984). Information richness: a new approach to managerial behavior and organizational design. Research in Organizational Behavior. 6: 191-233.

De Giorgi, G., Pellizzari, M., Redaelli, S. (2010). Identification of social interactions through partially overlapping peer groups. America Economic Journal: Applied Economic. 2(2): 241-275. 
De Melo, G. (2011). Peer effects identified through social networks: Evidence from Uruguayan schools. Working Paper, Department of Economics, University of Siena.

Duan, W., Gu, B., and Whinston, A.B. (2009). Informational cascades and software adoption on the Internet: An empirical investigation. MIS Quarterly. 33(1): 23-48.

Durlauf, S. N. and Young, H. P. (2001). The New Social Economics. In S. N. Durlauf and H. P. Young (eds), Social Dynamics. Cambridge, Massachusetts: MIT Press, pp. 1-14.

Faraj, S., von Krogh, G., Monteiro, E., and Lakhani, K.R. (2016). Special section introduction-Online community as space of knowledge flows. Information Systems Research. 27(4): 668-684.

Freeman, L. (1977). A set of measures of centrality based on betweenness. Sociometry. 40: 35-41.

Godes, D. and Mayzlin, D. (2004). Using online conversations to study word-of-mouth communication, Marketing Science. 23(4), 545-560.

Granovetter, M.S. (1973). The strength of weak ties. American Journal of Sociology. 78(6): 1360-1380.

Grant, R.M. (1996). Prospering in dynamically-competitive environments: Organizational capability as knowledge integration. Organization Science. 7(4): 375-387.

Hass, M.R., Criscuolo, P., and George, G. (2015). Which problems to solve? Online knowledge sharing and attention allocation in organizations. Academy of Management Journal. 58(3): 680-711.

Jackson, M.O. (2006). The economics of social networks. In Advances in economics and econometrics, theory and applications: Ninth world congress of the Econometric society. Vol. 1, ed. R. Blundell, W. Newey and T. Persson, Chapter 1. Cambridge University Press.

Jeppesen, L.B. and Frederiksen, L. (2006). Why do users contribute to firm-hosted user communities? The case of computer-controlled music instruments. Organization Science. 17(1): 45-63.

John, G. (2006). The essential impact of context on organizational behavior. The Academy of Management Review. 31(2): 386-408.

Kankanhalli, A., Tan, B.C.Y., Wei, K.K. (2005). Contributing knowledge to electronic knowledge repositories: an empirical investigation. MIS Quarterly. 29(1): 113-143.

Klein, K.J., Tosi, H., Cannella, A.A. (1999). Multilevel theory building: Benefits, barriers, and new developments. Academy of Management Review. 24(2): 243-248.

Landis, J.R., Koch, G.G. (1977). The measurement of observer agreement for categorical data. Biometrics 33 (1): 159-174.

Lave, J., Wenger, E. (1991) Situated learning: legitimate peripheral participation. Cambridge University Press: Cambridge.

Leary, M. R., Forsyth, D. R. (1987). Attributions of responsibility for collective endeavors. Review of Personality and Social Psychology. 8: 167-188.

Lee, L.F., (2003). Best spatial two-stage least squares estimators for a spatial autoregressive model with autoregressive disturbances. Econometric Reviews 22 (4): 307-335.

Majchrzak, A., Wagner, C., and Yates. D (2013). The impact of shaping on knowledge reuse for organizational improvement with Wikis. MIS Quarterly. 37(2): 455-470.

Manski, C. (1993). Identification of endogenous social effects: the reflection problem. Review of Economic Studies. 60: 531-542.

Manski, C. (2000). Economic analysis of social interactions. Journal of Economic Perspectives. 14(3): 115-136.

Markus, M.L., Steinfield, C.W., Wigand, R.T., and Minton, G. (2006). Industry-wide information systems standardization as collective action: the case of the U.S. residential mortgage industry. MIS Quarterly, 30 (Special Issue): 439-465. 
Moingeon, B., Quelin, B., Dalsace, F., Lumineau, F. (2006). Inter-organizational communities of practice: specificities and stakes. Working Paper, HEC Paris.

Mowday, R.T., Sutton, R.I. (1993). Organizational behavior: Liking individuals and groups to organizational contexts. Annu. Rev. Psychol. 44: 195-229.

Nelson, R.R., Winter, S.G. (1982). An evolutionary theory of economic change. Belknap Press of Harvard University: Cambridge, MA.

Nonaka, I. (1994). A dynamic theory of organizational knowledge creation. Organization Science. 5(1): 14-37.

Peddibhotla, N.B., Subramani, M.R. (2007). Contributing to public document repositories: A critical mass theory perspective. Organization Studies, 28. 327-346.

Perry, M. J. (2013). World stock market capitalization closes year at $\$ 54.6$ trillion. American Enterprise Institute. Retrieved from http://www.aei.org.

Peters, L.H., O’Connor, E.J., and Eulberg, J.R. (1985). Situational constraints: Sources, consequences, and future considerations. Research in Personnel and Human Resources Management. 3: 79-113.

Pool, V.K., Stoffman, N., Yokner, S. (2014). The people in your neighborhood: Social interactions and mutual fund portfolios. The Journal of Finance. Forthcoming.

Richard, L., Chiocchio, F., Essiembre, H., Tremblay, M., Lamy, G., Champagne, F., and Beaudet, N. (2014). Communities of Practice as a Professional and Organizational Development Strategy in Local Public Health Organizations in Quebec, Canada: An Evaluation Model. Healthc Policy, 9(3): 26-39.

Ridings, C., Gefen, D., Arinze, B. (2006). Psychological barriers: lurker and poster motivation and behavior in online communities, Communications of the Association for Information Systems 18: 329-354.

Roberts, J.A., Hann, I., and Slaughter, S.A. (2006). Understanding the motivations, participation, and performance of open source software developers: A longitudinal study of the Apache projects. Management Science. 52(7): 984-999.

Saez, E., Duflo, E., (2003). The role of information and social interactions in retirement plan decisions: Evidence from a randomized experiment. Quarterly Journal of Economics. 118: 815-842.

Sarker, S., Valacich, J.S. (2010). An alternative to methodological individualism: A non-reductionist approach to studying technology adoption by groups. MIS Quarterly. 34(4): 779-808.

Shah, S.K. (2006). Motivation, governance, and the viability of hybrid forms in open source software development. Management Science. 52(7): 1000-1014.

Shriver, S.K., Nair, H.S., Hofstetter, R. (2013). Social ties and user-generated content: Evidence from an online social network. Management Science. 59(6): 1425-1443.

Simon, H. (1991). Bounded rationality and organizational learning. Origination Science. 2: 125-134.

Soetevent, A. (2006). Empirics of the identification of social interactions: An evaluation of the approaches and their results. Journal of Economic Surveys, 20(2): 193 - 228.

Spaeth, S., von Krogh, G., and He. F. (2015). Perceived firm attributes and intrinsic motivation in sponsored open source software projects. Information Systems Research. 26(1): 224-237.

Sundararajan, A. (2008). Local network effects and complex network structure. The BE Journal of Theoretical Economics. 7(1): 1-37.

Trogdon, J., Nonnemaker, J., Pais, J., (2008). Peer effects in adolescent overweight. Journal of Health Economics. 27(5): 1388-1399. 
Tsai, H.T., Bagozzi, R.P. (2014). Contribution behavior in virtual communities: cognitive, emotional and social influences. MIS Quarterly. 38(1): 143-163.

Valente, T.W. (2005). Network models and methods for studying the diffusion of innovations. Models and methods in social network analysis (edited by Carrington, P.J., Scott, J., Wasserman, S.) Cambridge University Press.

Wasko, M.M., Faraj, S. (2005). Why should I share? Examining social capital and knowledge contribution in electronic networks of practice. MIS Quarterly. 29(1): 35-57.

Wenger E., McDermott R., Snyder W. (2002). Cultivating communities of practice. Harvard Business School Press: Boston.

Wiertz, C., Ruyter, K. (2007). Beyond the call of duty: Why customers participate in firm-hosted online communities. Organization Studies. 28, 349-378.

Wilson, J.M., O'Leary, M.B., Metiu, A., and Jett, Q.R. (2008). Perceived proximity in virtual work: Explaining the paradox of far-but-close. Organization Studies. 29(7): 979-1002.

Wuchty, S. (2009). What is a social tie? PNAS. 106(36): 15099-15100.

Xia M., Huang, Y., Duan, W., Whinston, A.B. (2012). To continue sharing or not to continue sharing? An empirical analysis of user decision in peer-to-peer sharing networks. Information Systems Research. 23(1): 247-259.

Zeng, X. and Wei, L. (2013). Social ties and user content generation: evidence from Flickr. Information Systems Research. 24(1): 71-87.

Zhang, X., Zhu, F. (2011). Group size and incentives to contribute: A natural experiment at Chinese Wikipedia. American Economic Review. 101(4): 1601-1615.

Zhao, K., Khan, S., Xia, M. (2011a). Sustainability of vertical standard consortia as communities of practice: A multi-level framework. International Journal of Electronic Commerce. 16(1): 11-40.

Zhao, K., Xia, M., Shaw, M.J. (2011b). What motivates firms to contribute to consortium-based ebusiness standardization? Journal of Management Information Systems. 28(2): 305-334.

Zheng, Y., Zhao, K., Stylianou, A. (2013). The impacts of information quality and system quality on users' continuance intention in information-exchange virtual communities: An empirical investigation. Decision Support Systems. 56: 513-524.

Figure 1: An Example of a Message Thread from the Virtual IOCoP

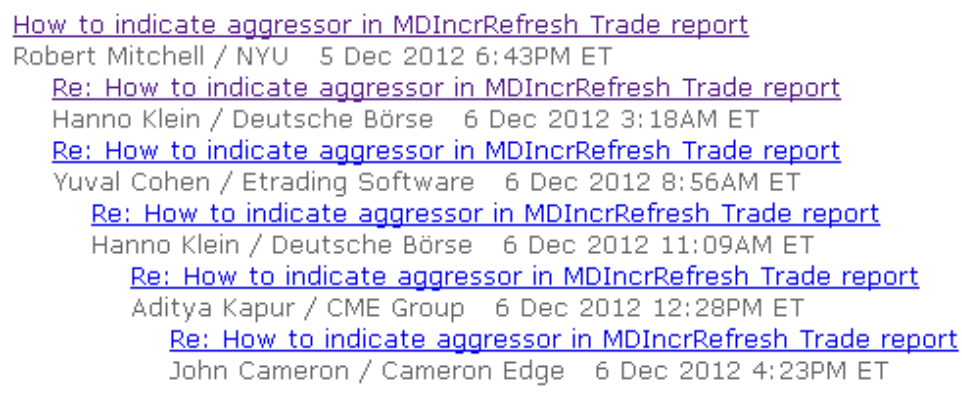


Figure 2: Illustration of IOCoP Conversation Network Construction

\begin{tabular}{|c|c|c|c|}
\hline $\begin{array}{l}A \\
P_{1}^{A} \\
1 \\
1 \\
1 \\
1 \\
v\end{array}$ & 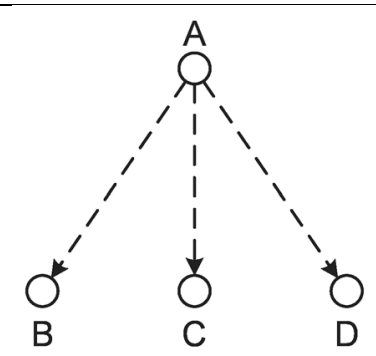 & $\stackrel{A}{\cap}$ & $\stackrel{A}{P}$ \\
\hline $\begin{array}{l}\text { (a) User A posts a question } \\
\text { in the IOCoP forum in time } \\
\text { period } t\end{array}$ & $\begin{array}{l}\text { (b) User B, C, and D read } \\
\text { A's question }\end{array}$ & $\begin{array}{l}\text { (c) B and D answer A's } \\
\text { question. C did not. }\end{array}$ & $\begin{array}{l}\text { (d) Connections (arcs) } \\
\mathrm{B} \rightarrow \mathrm{A} \text { and } \mathrm{D} \rightarrow \mathrm{A} \text { are } \\
\text { created. The arcs represent } \\
\text { communication between } \\
\text { users. }\end{array}$ \\
\hline
\end{tabular}

Figure 3: The IOCoP's Post Conversation Network in Year 2011

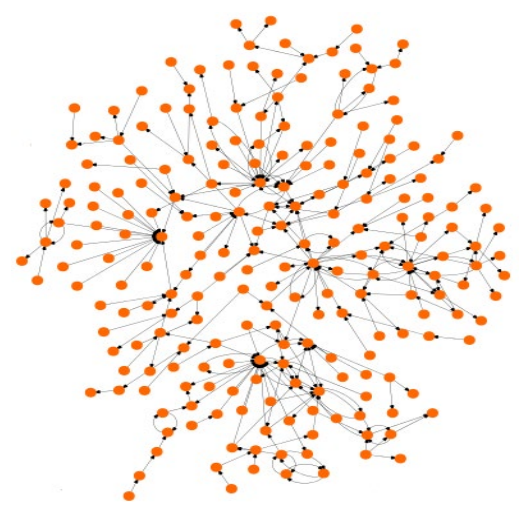

Table 1: Variable Descriptions and Summary Statistics

\begin{tabular}{|c|c|c|c|c|c|}
\hline & Variable & Mean & SD & Min & Max \\
\hline & \multicolumn{5}{|l|}{ Response Variable } \\
\hline $\mathbf{y}_{t \_Q U E S}$ & \# of questions ask by an individual in year $t$ & 0.995 & 1.735 & 0 & 29 \\
\hline $\mathbf{y}_{t \_A N S}$ & $\#$ of answers provided by an individual in year $t$ & 1.891 & 6.872 & 0 & 159 \\
\hline $\mathbf{y}_{t} H E L P$ & $\begin{array}{l}\text { average helpfulness of answers by an individual in year } t \\
\text { Individual Level Attributes }\end{array}$ & 1.715 & 1.343 & 0 & 4 \\
\hline $\mathbf{x}_{t_{-} \text {Tenure }}$ & $\begin{array}{l}\text { Tenure: number of years an individual has participated in } \\
\text { the IOCoP }\end{array}$ & 1.791 & 1.034 & 1 & 16 \\
\hline $\mathbf{x}_{\text {tInfo benefit }}$ & $\begin{array}{l}\text { Information benefit: average quality of answers an } \\
\text { individual receives in year } t\end{array}$ & 1.524 & 1.600 & 0 & 4 \\
\hline $\mathbf{x}_{\text {t_Degree }}$ & $\begin{array}{l}\text { Degree centrality: weighted number of unique peers a focal } \\
\text { individual is connected to in the conversation network }\end{array}$ & 3.517 & 5.768 & 0 & 91 \\
\hline \multirow[t]{2}{*}{$\mathbf{x}_{\text {t_One-timer }}$} & $\begin{array}{l}\text { One-timer: participants who only participate in current year } \\
(\text { yes }=1, \text { no }=0)\end{array}$ & 0.512 & 0.500 & 0 & 1 \\
\hline & Organizational Level Attributes & & & & \\
\hline
\end{tabular}




\begin{tabular}{|c|c|c|c|c|c|}
\hline $\mathbf{x}_{t \_ \text {Member }}$ & $\begin{array}{l}\text { Member: individuals whose organization is a member of the } \\
\text { consortium hosting the IOCoP } \\
\text { (yes }=1, \text { no }=0 \text { ) }\end{array}$ & 0.271 & 0.445 & 0 & 1 \\
\hline $\mathbf{x}_{\text {t_Org Knowledge }}$ & $\begin{array}{l}\text { Organizational Knowledge: total number of unique } \\
\text { individuals from the same organization answering questions } \\
\text { in the IOCoP in year } t\end{array}$ & 0.859 & 1.167 & 0 & 8 \\
\hline $\mathbf{x}_{t_{-} \text {Vendor }}$ & $\begin{array}{l}\text { IT Vendor: individuals whose organization is an IT vendor } \\
(\mathrm{yes}=1, \mathrm{no}=0)\end{array}$ & 0.395 & 0.489 & 0 & 1 \\
\hline \multirow[t]{2}{*}{$\mathbf{x}_{t_{-} \text {Sottware User }}$} & $\begin{array}{l}\text { Software user: individuals whose organization is a software } \\
\left.\text { user (yes }=1, \mathrm{no}^{\prime}=0\right)\end{array}$ & 0.356 & 0.479 & 0 & 1 \\
\hline & Aggregated Peer Contribution & & & & \\
\hline $\mathbf{x}_{t \_ \text {Aggr Peer }}$ & Total number of posts from all individuals in year $t$ & 602.7 & 133.9 & 412 & 905 \\
\hline
\end{tabular}

Table 2: Notations of the Econometric Model

\begin{tabular}{cl}
\hline$y_{i t}$ & Value of response variables of individual $i$ in year $t$. \\
$\mathbf{y}_{t}$ & An $n \times 1$ vector of response variable for all individual in year $t$. \\
$x_{i t}$ & An $1 \times K$ vector of the observable characteristics of individual $i$ in year $t$. \\
$\mathbf{X}_{t}$ & An $n \times K$ matrix of individual observable characteristics in year $t$. \\
$W_{j i t}$ & $W_{j i t}=1 / n_{i t}$ if individual $j$ responds to $i$ 's original post in year $t ; 0$ otherwise. \\
$n_{i t}$ & The total number of individuals responding to $i$ in year $t$ \\
$\mathbf{W}_{t}$ & An $n \times n$ matrix describing the post conversations between individuals \\
$\eta_{i}$ & Individual level fixed effects of individual $i$, correlated with $x_{i t}$. \\
$\boldsymbol{\eta}$ & An $n \times 1$ vector of the individual level fixed effects for all $n$ individuals. \\
$\beta$ & Coefficient of endogenous peer effects \\
$\gamma$ & Coefficient of participant's own characteristics \\
$\delta$ & Coefficient of exogenous peer effects \\
\hline
\end{tabular}

Table 3: Main Model Estimation Results

\begin{tabular}{|c|c|c|c|}
\hline Variables & $\begin{array}{c}\text { (1) } \\
\text { \# of Questions }\end{array}$ & $\begin{array}{c}\text { (2) } \\
\text { \# of Answers }\end{array}$ & $\begin{array}{c}\text { (3) } \\
\text { Helpfulness }\end{array}$ \\
\hline \multicolumn{4}{|l|}{ Own Characteristics } \\
\hline \multicolumn{4}{|l|}{ Individual Level } \\
\hline Tenure & $\begin{array}{r}-0.36 * * * \\
(0.12)\end{array}$ & $\begin{array}{r}0.24 * * * \\
(0.090)\end{array}$ & $\begin{array}{r}0.027 * * \\
(0.012)\end{array}$ \\
\hline Information benefit & $\begin{array}{c}0.16 * * * \\
(0.052)\end{array}$ & $\begin{array}{r}0.19 \\
(0.16)\end{array}$ & $\begin{array}{r}0.024 * * * \\
(0.0026)\end{array}$ \\
\hline Degree centrality & $\begin{array}{c}0.14^{* * *} \\
(0.050)\end{array}$ & $\begin{array}{r}0.70 * * \\
(0.31)\end{array}$ & $\begin{array}{r}0.019 * * * \\
(0.0036)\end{array}$ \\
\hline One-timer & $\begin{array}{r}0.49 * * * \\
(0.063)\end{array}$ & $\begin{array}{r}0.078 * * * \\
(0.022)\end{array}$ & $\begin{array}{r}0.23 * * * \\
(0.052)\end{array}$ \\
\hline Organizational Level & & & \\
\hline
\end{tabular}




\begin{tabular}{|c|c|c|c|}
\hline Member & $\begin{array}{r}-0.25 * * * \\
(0.064)\end{array}$ & $\begin{array}{r}1.88 * * * \\
(0.24)\end{array}$ & $\begin{array}{r}1.24 * * * \\
(0.42)\end{array}$ \\
\hline Organizational Knowledge & $\begin{array}{r}-0.13 * * * \\
(0.042)\end{array}$ & $\begin{array}{r}-0.34 * * * \\
(0.11)\end{array}$ & $\begin{array}{r}0.20 * * * \\
(0.025)\end{array}$ \\
\hline IT Vendor & $\begin{array}{r}-0.49 * * \\
(0.22)\end{array}$ & $\begin{array}{r}1.05 * * * \\
(0.36)\end{array}$ & $\begin{array}{r}0.063 * * \\
(0.021)\end{array}$ \\
\hline Software user & $\begin{array}{r}0.99 \\
(0.91)\end{array}$ & $\begin{array}{r}-1.43 * * * \\
(0.36)\end{array}$ & $\begin{array}{r}-0.012 * * * \\
(0.0046)\end{array}$ \\
\hline Endogenous peer effects & $\begin{array}{r}0.30 * * \\
(0.13) \\
\end{array}$ & $\begin{array}{r}0.38 * * \\
(0.16) \\
\end{array}$ & $\begin{array}{r}0.69 * * * \\
(0.26)\end{array}$ \\
\hline \multicolumn{4}{|l|}{ Exogenous peer effects } \\
\hline \multicolumn{4}{|l|}{ Individual Level } \\
\hline Tenure & $\begin{array}{r}0.032 * * * \\
(0.012)\end{array}$ & $\begin{array}{r}0.056 * * * \\
(0.010)\end{array}$ & $\begin{array}{r}0.032 * * \\
(0.014)\end{array}$ \\
\hline Information Benefit & $\begin{array}{r}0.030 \\
(0.021)\end{array}$ & $\begin{array}{r}0.080 \\
(0.051)\end{array}$ & $\begin{array}{r}0.16^{* * *} \\
(0.025)\end{array}$ \\
\hline Degree centrality & $\begin{array}{r}0.010 \\
(0.0072)\end{array}$ & $\begin{array}{r}0.043 * * * \\
(0.013)\end{array}$ & $\begin{array}{r}-0.039 * * * \\
(0.010)\end{array}$ \\
\hline One-timer & $\begin{array}{r}0.014 \\
(0.010)\end{array}$ & $\begin{array}{r}0.12 \\
(0.084)\end{array}$ & $\begin{array}{r}0.049 \\
(0.035)\end{array}$ \\
\hline \multicolumn{4}{|l|}{ Organizational Level } \\
\hline Member & $\begin{array}{r}0.041 * * * \\
(0.012)\end{array}$ & $\begin{array}{r}0.16 \\
(0.15)\end{array}$ & $\begin{array}{r}0.070 * * \\
(0.032)\end{array}$ \\
\hline Organizational Knowledge & $\begin{array}{r}0.065 * * * \\
(0.018)\end{array}$ & $\begin{array}{r}0.038 \\
(0.028)\end{array}$ & $\begin{array}{r}0.0047 \\
(0.0035)\end{array}$ \\
\hline IT Vendor & $\begin{array}{r}-0.17 \\
(0.11)\end{array}$ & $\begin{array}{r}0.26^{* *} \\
(0.12)\end{array}$ & $\begin{array}{r}0.048 * * \\
(0.023)\end{array}$ \\
\hline Software user & $\begin{array}{r}0.022 \\
(0.015)\end{array}$ & $\begin{array}{r}0.51 * * \\
(0.23)\end{array}$ & $\begin{array}{r}-0.024 \\
(0.016) \\
\end{array}$ \\
\hline \multicolumn{4}{|l|}{ Homophily } \\
\hline Post time & $\begin{array}{r}0.017 \\
(0.012)\end{array}$ & $\begin{array}{r}0.024 \\
(0.019)\end{array}$ & $\begin{array}{r}0.021 \\
(0.016)\end{array}$ \\
\hline Social circle & $\begin{array}{r}0.044 \\
(0.028) \\
\end{array}$ & $\begin{array}{r}0.062 \\
(0.042) \\
\end{array}$ & $\begin{array}{r}0.018 \\
(0.013) \\
\end{array}$ \\
\hline $\begin{array}{l}\text { Year fixed effects } \\
\text { Aggregated peer contribution }\end{array}$ & $\begin{array}{r}0.0042 \\
(0.0029)\end{array}$ & $\begin{array}{r}0.0039 \\
(0.011)\end{array}$ & $\begin{array}{l}0.0092 \\
(0.014) \\
\end{array}$ \\
\hline Observations & 2,024 & 2,024 & 2,024 \\
\hline Adj. $R^{2}$ & 0.44 & 0.42 & 0.66 \\
\hline
\end{tabular}

Table 4. The network position type change of control and treatment groups over time 


\begin{tabular}{|l|l|c|c|}
\hline & Size & Start of time period & End of time period \\
\hline Control group & 200 & Type $1 \bullet$ & Type 1 \\
\hline Treatment & 200 & Type $1 \bullet$ & Type 2 \\
\hline
\end{tabular}

Table 5. Average treatment effect of Peer Influences on purchase of individuals in control and treatment groups over time

\begin{tabular}{|l|c|}
\hline & PSM \\
\hline Treatment vs. Control, & $0.16^{* *}$ \\
questions asked & $(0.061)$ \\
\hline Treatment vs. Control, & $0.27^{* *}$ \\
questions answered & $(0.10)$ \\
\hline Treatment vs. Control, & $0.24^{*}$ \\
helpfulness & $(0.12)$ \\
\hline Note. Data above demonstrate the mean \\
and standard deviation (in parentheses) of \\
average treatment effects. \\
$*^{*}: p<0.01 ; *: p<0.05$. \\
\hline
\end{tabular}

Table 6: An Alternative Model Specification

\begin{tabular}{|c|c|c|c|}
\hline Model & (1) & (2) & (3) \\
\hline Variables & \# of questions & \# of answers & Helpfulness \\
\hline \multicolumn{4}{|l|}{ Own Characteristics } \\
\hline \multicolumn{4}{|l|}{ Individual Level } \\
\hline \multirow[t]{2}{*}{ Tenure } & $-0.47 * *$ & $0.31 * * *$ & $0.030 * *$ \\
\hline & $(0.21)$ & $(0.082)$ & $(0.013)$ \\
\hline \multirow[t]{2}{*}{ Information benefit } & $0.18 * * *$ & 0.40 & $0.015 * *$ \\
\hline & $(0.041)$ & $(0.30)$ & $(0.0074)$ \\
\hline \multirow[t]{2}{*}{ Degree centrality } & $0.14 * * *$ & $0.52 * *$ & $0.015 * * *$ \\
\hline & $(0.0064)$ & $(0.26)$ & $(0.0036)$ \\
\hline \multirow[t]{2}{*}{ One-timer } & $0.40 * * *$ & $0.072 * *$ & $0.26 * * *$ \\
\hline & $(0.079)$ & $(0.035)$ & $(0.037)$ \\
\hline \multicolumn{4}{|l|}{ Organizational Level } \\
\hline \multirow[t]{2}{*}{ Member } & $-0.22 * *$ & $1.9 * * *$ & $0.78^{* *}$ \\
\hline & $(0.092)$ & $(0.35)$ & $(0.36)$ \\
\hline \multirow[t]{2}{*}{ Organizational knowledge } & $-0.083 * * *$ & $-0.17 * * *$ & $0.25 * * *$ \\
\hline & $(0.024)$ & $(0.057)$ & $(0.076)$ \\
\hline \multirow[t]{2}{*}{ IT Vendor } & $-0.53 * * *$ & $1.4 * * *$ & $0.043 * * *$ \\
\hline & $(0.20)$ & $(0.40)$ & $(0.017)$ \\
\hline \multirow[t]{2}{*}{ Software user } & 1.1 & $-1.6^{* * *}$ & $-0.019 * * *$ \\
\hline & $(0.81)$ & $(0.38)$ & $(0.0053)$ \\
\hline \multirow[t]{2}{*}{ Endogenous peer effect } & $0.37 * * *$ & $0.41 * * *$ & $0.50 * *$ \\
\hline & $(0.096)$ & $(0.090)$ & $(0.24)$ \\
\hline Year fixed effects & 0.0064 & 0.0045 & 0.0084 \\
\hline Aggregated peer contribution & $(0.0084)$ & $(0.0048)$ & $(0.012)$ \\
\hline Observations & 2,024 & 2,024 & 2,024 \\
\hline
\end{tabular}




\begin{tabular}{|c|c|c|c|}
\hline Adj. $R^{2}$ & 0.41 & 0.31 & 0.63 \\
\hline
\end{tabular}
$p<0.01, * * p<0.05, * p<0.10$, standard errors in parentheses 